\title{
Factorial Design Executed Development of Miconazole Nitrate Microemulsion Based Bioadhesive Gel and Its Evaluation
}

\author{
Abhirami Venkatachalam ${ }^{1}$, Harini Chowdary Vadlamudi ${ }^{2}$, Sneha Bharti ${ }^{3}$ \\ ${ }^{1}$ Senior Data Coordinator, Data Reconciliation Center, Labcorp Drug Development, Bengaluru. \\ ${ }^{2}$ Senior RWD Analyst, RWDMP, GSK, Bengaluru. \\ ${ }^{3}$ Assistant Professor, Kashi Institute of Pharmacy, Mirzamurad, Varanasi, U.P. 221307 \\ Corresponding Author: Harini Chowdary Vadlamudi
}

DOI: https://doi.org/10.52403/ijrr.20220167

\section{ABSTRACT}

Miconazole nitrate $(\mathrm{MN})$ is a poorly aqueous soluble antifungal drug. The microemulsion (ME) based bioadhesive antifungal gel of MN was designed to improve the antifungal activity by increasing the bioadhesion potential. Based on the solubility results, components of $\mathrm{ME}$ were selected viz. rice bran oil: tween 80: propylene glycol and proceeded with pseudo-ternary phase diagram studies. $2^{3}$ factorial design was employed for the formulation of MEs employing water titration technique. The MEs was examined for further studies and was optimized using DESIGN EXPERT 12 software considering the responses like globule size, zeta potential and in vitro drug release. Tamarind seed polysaccharide (TSP) and carbopol 934 were used as gel bases for the formulation of microemulsion-based bioadhesive gels. The gels were examined for drug content, $\mathrm{pH}$, spreadability, viscosity, bioadhesive strength, ex vivo drug permeation and in vivo skin irritation potential. The antifungal activity against Candida albicans and Cryptococcus neoformans of four formulations (MN-ME, MNME based TSP gel, MN- ME based carbopol gel and marketed miconazole gel (2\% Miconazole gel) was evaluated using modified agar diffusion method. The results revealed that microemulsion based bioadhesive gel (MBG) of TSP and CP exhibited drug content of about $87.29 \%$ and $83.34 \%$ and ex vivo skin permeation of $84.21 \%$ and $73.94 \%$ at the end of $10 \mathrm{~h}$. MBG of TSP showed better antifungal
\end{abstract}

activity and no skin irritation potential in comparison to the carbopol gel. Therefore, MBG of TSP has the ability to enhance the contact time owing to greater bioadhesion thereby providing a better therapeutic activity.

Key Words: Miconazole nitrate, Tamarind Gum, Topical delivery, Microemulsion based bioadhesive gel, fungal infection.

\section{INTRODUCTION}

Microbial invasion into the skin is the main reason for skin infections. Microbes generally remains in dormant state and feed off the dead skin cells. However, due to non hygenic habits, the fungi reproduce quickly, resulting in an infection. Candidiasis, Athlete's foot and ringworms are a few of the general skin conditions. Fungal conditions occurs more frequently in immuno suppressed patients with diabetics, HIV infected patients, individuals on steroids or cancer treatment, etc [1]. The incidence of fungal infections of skin is escalating throughout the world. Around 40 million people have been suffering from fungal infections. With the fungal infections immune system functioning will get hindered which makes a promising progression in the disease. Dermatophytes and candidal infections are the most frequent fungal infections affecting skin. Topical antifungal agents have been administered as antifungal 
ointments, creams, gels, sprays and lotions. In addition, antifungal medication can be given orally and IV route. Some frequent antifungal prescription medicines are clotrimazole, econazole, miconazole, terbinafine, fluconazole, ketoconazole, amphotericin, echinocandins and flucytosine [2].

Traditional drug therapy due to its numerous advantages like, targeting the infection site, diminution of the systemic side effects, enhancement of the therapeutic efficacy and, better patient compliance; it is most widely used for the delivery of the drug into the body [3]. Topical application of medicine provides advanced local tissue levels, fast drug delivery, and lesser systemic exposure in comparison to oral administration [4]. Topically applied molecule may penetrate either by the intercellular path of the stratum corneum lipid bilayers, or by the transcellular path via the corneocytes. Hair follicles and related sebaceous glands and sweat glands are possible route by which the drug compound may get into the skin however, it accounts for only $0.1 \%$ of the total skin surface area. Drug molecules may effectively diffuse through the stratum corneum layer provided they have reasonably small having the size of about 500 Da, lipophilic in nature $(\log \mathrm{P} 1-3)$ and water soluble [5]. Conventional drug delivery system has drawbacks such as short contact time with the oral mucosa consequencing frequent administration every day [6]. Microemulsions (ME) generally are clear, monophasic colloidal dispersions composed of oil, water, surfactant and cosurfactant having the droplet sizes in the range 10-100 $\mathrm{nm}$ [7]. ME having low interfacial energy are anticipated to potentially brake by temperature changes and/or dilution [8]. The major physicochemical properties of ME include transparency, optical isotropy, low viscosity, and thermodynamic stability. ME have quite a lot of advantages for instance easy and simple preparation, stability, improved solubility of drug, controlled drug delivery, and better bioavailability of both hydrophilic and lipophilic drugs through various routes. The components of $\mathrm{ME}$ act as penetration enhancers that might ease the diffusional barrier of the stratum corneum and boost drug permeation rate and absorption via the skin. The permeability of drug formulations through the stratum corneum may be influenced by the hydration effect of ME [9].

Microemulsions are difficult to stabilize on the skin surface because of low viscosity. In order to overcome this problem, microemulsion based gel formulations are developed using gelling agents as a base to incorporate microemulsions. These types of formulations combine the advantages of microemulsions and gels. Therefore, it shows potential for effective transdermal and dermal drug administration. MEs or ME gels significantly enhance the absorption of drugs compared with solution, gel or cream formulations [10]. ME-based gels (MBGs) are known to provide faster drug release than conventional formulations [11]. Gels can be described as semi rigid systems wherein the movement of the dispersing medium is confined by an interweaving 3D system of particles or solvated macromolecules in the dispersed phase. Gels are relatively stable, controls the drug release from its structure and better absorption along with bioavailability compared to other semisolid dosage forms [12] (Loftsson, 2014). Therefore, in order to overcome these problems microemulsion based bioadhesive gels is being designed to combine the advantages of microemulsions and gels.

Miconazole nitrate is a synthetic antifungal imidazole that has been used effectively and safely for nearly 40 years to treat superficial fungal infections [13]. Miconazole may harm fungal cell membrane integrity, change fungal attachment, and inhibit germ tube and mycelia formation by interacting with 
cytochrome p450 complex to inhibit ergosterol biosynthesis [14]. Miconazole has a powerful wide range of activities against many species of Candida, including Candida albicans [15], Candida glabrata [16], Candida dubliniensis, Candida parapsilosis and Candida tropicalis [17]. With this context the present study was designed for the development and investigation of miconazole nitrate microemulsion based bioadhesive gel using tamarind seed polysaccharide (TSP) and carbopol 934 (CP) as gel bases to counteract topical fungal infections.

\section{MATERIALS}

Miconazole nitrate (MN) was obtained from Yarrow Chem Products, Mumbai, India., Coconut oil (CO) from Karnataka Fine Chem., Bengaluru, Oleic acid (OA) from $\mathrm{CDH}$, New Delhi., and Rice bran oil (RBO), Sunflower oil (SO), Sesame oil (SO), Olive oil (OO), Palm oil (PO), were purchased from local vendors; surfactants like Tween 80, Tween 20, and co-surfactants including Propylene glycol (PG), Glycerol, and Polyethylene glycol 400 (PEG 400) were procured from Karnataka Fine Chem., Bengaluru. Span 80, Span 60 and Span 20 were procured from CDH, New Delhi. Carbopol 934P were procured from Yarrow Chem Products, Mumbai. Muller hington media was obtained from HiMedia, Mumbai, India. All the other chemicals used in the formulation were of analytical reagent grade.

\section{METHODS}

\subsection{Screening of ME components}

For screening of components to be incorporated into $\mathrm{ME}$, the saturated solubility of $\mathrm{MN}$ in various oils such as (Rice Bran Oil, Sunflower Oil, Olive Oil, Coconut Oil, Sesame Oil, Palm Oil and Oleic Acid), surfactant (Tween 80, Tween 20, span 80, span 60 and span 20) and cosurfactant (PG, glycerol, and PEG 400) was determined. $1 \mathrm{mg}$ of miconazole nitrate was taken in a test tube containing $10 \mathrm{ml}$ of oil or surfactant or co surfactant separately and the dispersion was mixed for $5 \mathrm{~min}$. The resulting mixtures were shaken reciprocally for $24 \mathrm{~h}$ at $37 \pm 0.5^{\circ} \mathrm{C}$ to reach equipoise. The supernatant were collected after filtering the mixture through a membrane filter paper $(0.45 \mu \mathrm{m})$. The amount of the $\mathrm{MN}$ in the obtained filtrate of each component were analyzed after diluting to an appropriate concentration with methanol using UV-Visible spectrophotometer (UV- 1700, Shimadzu, Japan) at $272 \mathrm{~nm}$. The components that showed maximum solubility of $\mathrm{MN}$ were selected to prepare MEs [9].

\subsection{Construction of Pseudo ternary phase diagram}

Pseudo ternary phase diagrams were constructed using water titration method to know the existence of $\mathrm{ME}$ range. The concentration range of components and the effect of surfactant/co surfactant weight ratios on the existence of stable ME regions were obtained. Three different phase diagrams were constructed with the 1:1, 2:1 and 3:1 weight ratios of tween 80 to $\mathrm{PG}$ respectively to select the ratio of surfactant and co surfactant. At selected surfactant cosurfactant ratio, the blend consisting of surfactant, propylene glycol as a co-surfactant, and oil was formulated with the volume ratio of oil to Smix at 9:1, 8:2, 7:3, 6:4, 5:5, 4:6, 3:7, 2:8, and $1: 9$, respectively to cover maximum ratios to know the boundaries of phases formed in the phase diagrams. The ME was titrated by drop wise addition of distilled water with continuous agitation at $37 \pm 1{ }^{\circ} \mathrm{C}$. The point where the $\mathrm{ME}$ mixture became clear and transparent was taken as the end point. The pseudoternary phase diagrams were obtained by calculating the concentration of oil, surfactant, co-surfactant, and water at suitable weight ratios to select component ratios required to prepare stable MEs [18].

\subsection{Microemulsion preparation}

According to the ME regions in the phase diagrams, eight formulations were 
selected at different component ratios for further study. The various ME formulations were prepared using the process of water titration method by changing the proportions of oil, surfactant and Smix ratio as depicted in Table 1 [19]. Briefly, MN was added into $100 \mathrm{ml}$ beaker to which appropriate amount oil, surfactant and propylene glycol as a cosurfactant was added followed by drop wise addition of water under magnetic stirring at $37{ }^{\circ} \mathrm{C}$ for the preparation of MN loaded MEs. The blend was further homogenized at $3500 \mathrm{rpm}$ to obtain a homogeneous solution. The final concentration of $\mathrm{MN}$ in microemulsion systems was $1 \%(\mathrm{w} / \mathrm{w})$.

Table 1: Composition of microemulsions

\begin{tabular}{|l|l|l|l|}
\hline Formulation code & Oil & Surfactant & Ratio \\
\hline F1 & Rice bran oil & Span 20 & $1: 1$ \\
\hline F2 & Rice bran oil & Tween 80 & $1: 1$ \\
\hline F3 & Rice bran oil & Span 20 & $1: 2$ \\
\hline F4 & Rice bran oil & Tween 80 & $1: 2$ \\
\hline F5 & Sunflower oil & Span 20 & $1: 1$ \\
\hline F6 & Sunflower oil & Tween 80 & $1: 1$ \\
\hline F7 & Sunflower oil & Span 20 & $1: 2$ \\
\hline F8 & Sunflower oil & Tween 80 & $1: 2$ \\
\hline
\end{tabular}

\subsection{Characterization of drug loaded Microemulsion}

The eight formulations of microemulsions were prepared and evaluated further for optimization.

\subsection{1. $\mathrm{pH}$ and Viscosity determination:}

The $\mathrm{pH}$ of all the prepared MEs (10\% solution) was evaluated using a digital $\mathrm{pH}$ meter ( $\mathrm{pH}$ Meter 115, Bengaluru) [20]. The viscosity of MEs was examined by means of Brookfield viscometer (DVII+Pro EXTRA) at the room temperature. Spindle number 61 was dipped in ME and rotated at $50 \mathrm{rpm}$ [21].

\subsubsection{Average droplet size, poly dispersity index and zeta potential determination:}

The measurement of average globule size, PDI and zeta potential of the MEs were calculated using Malvern Zetasizer at $25^{\circ} \mathrm{C}$ (Nano ZS90, Instruments, UK) [22,23].

\subsubsection{Drug content studies:}

Drug content of $1 \%$ MEs in methanol was evaluated using UV spectrophotometer (DV-11+ pro EXTRA). The resulting solution was then centrifuged at $3500 \mathrm{rpm}$ for $15 \mathrm{~min}$ and filtered using $0.45 \mu \mathrm{m}$. The absorbance was determined spectrophotometrically at $272 \mathrm{~nm}$ as demonstrated by Kailas. [10].

\subsubsection{Transmittance measurement}

Transparency of prepared MEs was measured by UV-visible spectrophotometer (Agilent technology Cary $60 \mathrm{UV}-\mathrm{Vis}, \mathrm{USA}$ ) at $272 \mathrm{~nm}$ using Phosphate buffer (pH 7.4) as blank [10].

\subsubsection{In vitro diffusion studies:}

Diffusion study of MEs was performed using cellophane membrane of $0.45 \mu \mathrm{m}$ pore size which was dispersed overnight in Phosphate buffer of $\mathrm{pH}$ 7.4. Freshly prepared phosphate buffer with pH 7.4 was used as a medium. The cellophane membrane was fixed to one end of the diffusion tube to cover it completely which is exclusively designed to be opened at both ends and was immersed in $200 \mathrm{ml}$ of diffusion media. Approximately $5 \mathrm{ml}$ of $\mathrm{ME}$ was positioned above the cellophane membrane of the cylinder. The complete assembly was kept under magnetic stirring to ensure continuous stirring of solution and is maintained at $37 \pm 0.5^{\circ} \mathrm{C}$. The stirring speed was adjusted such that no vortex was formed. About $5 \mathrm{ml}$ of aliquot was taken at determined time interval (1h) for up to $8 \mathrm{~h}$ by replacing it with equal amount of fresh diffusion media to maintain the sink condition and is equilibrated to $37 \pm 0.5^{\circ} \mathrm{C}$. All samples were filtered through Whatman filter paper. The concentration of $\mathrm{MN}$ in each aliquot was examined and recorded by using Agilent technologies Cary-60 UV-Visible spectrophotometer at $272 \mathrm{~nm}$ [10]. 


\subsection{Optimization}

A factorial design of $2^{3}$ was applied to the formulation, with 3 factors at 2 levels giving a total of 8 runs. The independent variables were oil, surfactant cosurfactant mixture and surfactant cosurfactant ratio. The obtained MEs were examined based on particle size, zeta potential and drug release kinetics. The responses obtained for the formulation related to particle size, zeta potential and drug release were statistically evaluated using Design-Expert $\mathrm{R} \quad 12.0$ software and design space was obtained for the development of optimized ME which was further utilized for formulation of microemulsion based bioadhesive gel.

\subsection{Formulation of microemulsion based bioadhesive gel}

The preservative blend of methyl and propyl paraben (3:1) was dissolved in hot water under stirring. The carbomer (Carbopol 934) and tamarind seed polysaccharide (TSP) was dispersed separately in a warm aqueous solution of parabens with constant stirring using magnetic stirrer at $2000 \mathrm{rpm}$. Miconazole nitrate MEs were incorporated into carbopol and TSP gel. The $\mathrm{pH}$ of carbopol and TSP gel was adjusted using Triethanolamine and borax. The composition of the final $\mathrm{MBG}$ was $1 \%$ (w/w) MN, 20\% (w/w) propylene glycol, $1 \% \quad(\mathrm{w} / \mathrm{w})$ gelling agent, $0.1 \% \quad(\mathrm{w} / \mathrm{w})$ mixture of methyl- and propyl paraben (3:1), and water. The prepared MBGs were subjected for various studies [24].

\subsection{Characterization and evaluation of drug loaded MBG}

\subsubsection{Determination of $\mathbf{p H}$ and Viscosity:}

The $\mathrm{pH}$ range of an ideal topical gel is found to be 4.5-7. The $\mathrm{pH}$ of the formed gels was determined using digital $\mathrm{pH}$ meter. Viscosity determination of the gel was done similarly manner as of microemulsion using $\mathrm{T}$ shape spindle no F96 and viscosity was recorded in cps. The viscosity of $\mathrm{MBG}$ was examined by means of Brookfield viscometer (DV-
II+Pro EXTRA) at the room temperature $[25,26]$.

\subsubsection{Average droplet size, PDI, zeta potential and Transmittance measurement:}

The measurement of average globule size, PDI and zeta potential of the MBG were calculated using Malvern Zetasizer at $25^{\circ} \mathrm{C}$ (Nano ZS90, Instruments, UK). Transparency of prepared MBG was measured by UV-visible spectrophotometer (Agilent technology Cary 60 UV-Vis, USA) at $272 \mathrm{~nm}$ using Phosphate buffer (pH 7.4) as blank.

\subsubsection{Drug content:}

$1 \mathrm{gm}$ of MBG was diluted to $10 \mathrm{ml}$ using methanol and sonicated for $10 \mathrm{~min}$. The solution then was filtered using $0.45 \mu \mathrm{m}$ and centrifuged at $3500 \mathrm{rpm}$ for $15 \mathrm{~min}$. The absorbance of the resulting solution was determined spectrophotometrically at $272 \mathrm{~nm}$.

\subsubsection{Spreadability:}

Spreadability is the time taken in seconds by the slides to slip off from the formulation or travel the distance of $5 \mathrm{~cm}$ under the application of a certain weight. About $1 \mathrm{~g}$ of MBG was sandwiched between the two glass slides each of $7.5 \mathrm{~cm}$ and a $20 \mathrm{~g}$ weight was attached to the upper glass plate and held in the vertical position. The time taken for the upper slide to slip off was noted and spreadability coefficient (S) was determined. Lesser the time taken for the separation of slides better is the spreadability [27].

$$
S=m \times \frac{l}{t}
$$

$\mathrm{m}=$ Weight tied to upper plate. $1=$ Length of glass plate.

$\mathrm{t}=$ Time taken in sec. 


\subsubsection{Ex vivo bioadhesive strength:}

Rat skin was separated and underlying fat and loose tissues were removed. The membrane was washed initially with distilled water followed by phosphate buffer $\mathrm{pH}$ 7.4. The fresh skin was cut into equal pieces and were tied to the two glass slide separately out of which one glass slide was fixed to the wooden piece (i,e., fixed portion) and other piece was tied with balance on right hand side. The right and left pans were balanced by adding extra weight on left hand pan. $1 \mathrm{~g}$ of MBG was placed between these two slides containing hairless skin pieces and extra weight from left pan was removed to sandwich two pieces of skin and some pressure was applied to remove the presence of air and this will improve the bioadhesion. Balance was kept in this position for $5 \mathrm{~min}$. Weight was added slowly at $100 \mathrm{mg} / \mathrm{min}$ to the left hand pan until patch detached from skin surface. The weight in gram required to detach the gel from skin surface gave the measure of bioadhesive strength [28].

Bioadhesive strength was calculated by using following:

$$
B_{S}=\frac{W_{g}}{A}
$$

Where, Bs is bioadhesive strength, $\mathrm{Wg}$ is weight required (in $\mathrm{g}$ ) and $\mathrm{A}$ is area (in $\mathrm{cm}^{2}$ )

\subsubsection{Ex vivo skin permeation studies:}

Ex vivo skin permeation studies was carried out using diffusion tube with an effective diffusion area of $2 \mathrm{~cm}^{2}$. The dorsal side of rat skin was excised and the membrane was washed with distilled water and then with phosphate buffer $\mathrm{pH}$ 7.4. The membrane was tied to the donor of diffusion tube with the stratum corneum facing the donor compartment. $1 \mathrm{~g}$ of $\mathrm{MBG}$ was applied on the donor compartment. The receptor compartment was filled with 200 $\mathrm{ml}$ of PBS pH 7.4 and maintained at $37^{\circ} \mathrm{C}$ with stirring at $100 \mathrm{rpm}$. At predetermined time intervals of $1 \mathrm{~h}$ up to $8 \mathrm{~h}, 5 \mathrm{ml}$ of aliquot was taken and replaced with the fresh diffusion media to matintain the sink conditions.

All samples were filtered through Whatman filter paper and analyzed spectrophotometrically at $272 \mathrm{~nm}$ as demonstrated by Sabale and Vora [29]. MN steady state flux permeation rate (Jss) was calculated by dividing slope of graph linear portion with diffusion cell area in $\mathrm{mg} / \mathrm{cm} 2 \mathrm{~min}$, a permeability coefficient $(\mathrm{Kp})$ was obtained by dividing Jss by the initial concentration of $\mathrm{MN}$ in donor chamber in $\mathrm{cm} 2 \mathrm{~min}-1$. Enhancement ratio (Er) was determined as the ratio of Jss to that of commercial gel. [30]

\subsubsection{Skin irritation study:}

The MBG formulations were examined for skin irritation potential as the formulation was intended for topical application. Skin irritation tests were performed on six Albino wistar rats which were grouped into two groups to determine irritancy after single application of MBG. To one group MBG of TSP was applied to the right side and left side was kept as control, whereas to the other group $\mathrm{CP}$ MBG was applied to the right side and to the left side marketed miconazole gel was applied which served as standard. The MBG and commercial $\mathrm{MN}$ gel was carefully applied on the depilated part of the rat skin and then the applied area was covered with gauze and adhesive bandage. The gauze was removed from rats and the exposed skin was graded for formation of edema and erythema. Scoring was recorded for a period of 7 days. Based on the observations on the skin, scores are given as per the Draize scoring system [31].

\subsubsection{Antifungal Study:}

The antifungal activity of formulations (MN-ME, MN-MBG of TSP and marketed miconazole gel) was evaluated against Candida albicans and Cryptococcus neoformans fungal strains adopting modified agar diffusion method. Candida albicans and Clostridium neoformans species were cultured in Muller 
hington media at $30^{\circ} \mathrm{C}$ for 2 days. Wells or holes were made using a metal borer and sterile discs were placed into the well, and every well was packed with a sufficient sample. All the plates were incubated for 10 days at $30^{\circ} \mathrm{C}$. Afterwards, the diameters of inhibition zones were calculated and noted. The experimentation was carried out in triplicate [32].

$$
\% \text { inhibition }=\frac{l_{2}}{l_{1}} \times 100
$$

Where $l_{1}$ is the total length of the streaked culture, and $l_{2}$ is the length of inhibition [33].

\subsubsection{Stability studies:}

Drug stability is the stability of a particular formulation, to remain within its physical, chemical, therapeutic and toxicological specifications throughout its shelf life in a specific container. The prepared optimised MBG of $\mathrm{MN}$ was packed in tightly closed container and kept away from light in stability chamber which is maintained at $40 \pm 2{ }^{\circ} \mathrm{C}$ and $75 \pm 5 \% \mathrm{RH}$ and $4{ }^{\circ} \mathrm{C}$ to evaluate the thermodynamic Stability. The optimized formulation was withdrawn and analysed at the end of 30th day for physical appearance, $\mathrm{pH}$, drug content, spreadability, viscosity, drug content and in vitro release study [23].

\section{RESULTS AND DISCUSSION}

4.1. Screening of Oils, Surfactants, and Co-surfactants for Microemulsion

Figure 1 reveals solubility of $\mathrm{MN}$ in various solvents such as oils, surfactants and co-surfactants at room temperature. Highsolubility solvents were prioritized. $\mathrm{MN}$ showed the highest solubility in rice bran oil followed by sunflower oil. Among surfactants, the solubility of the drug in Span 20, Span 80 and Span 60. The aforementioned two components (ie Span 80 and Span 60) are compatible, but they did not form a stable microemulsion during the study. The second high- solubility surfactant Tween 80, formed a stable microemulsion. Among cosurfactants, the solubility of the $\mathrm{MN}$ in propylene glycol was greater than that in glycerin and PEG400 .

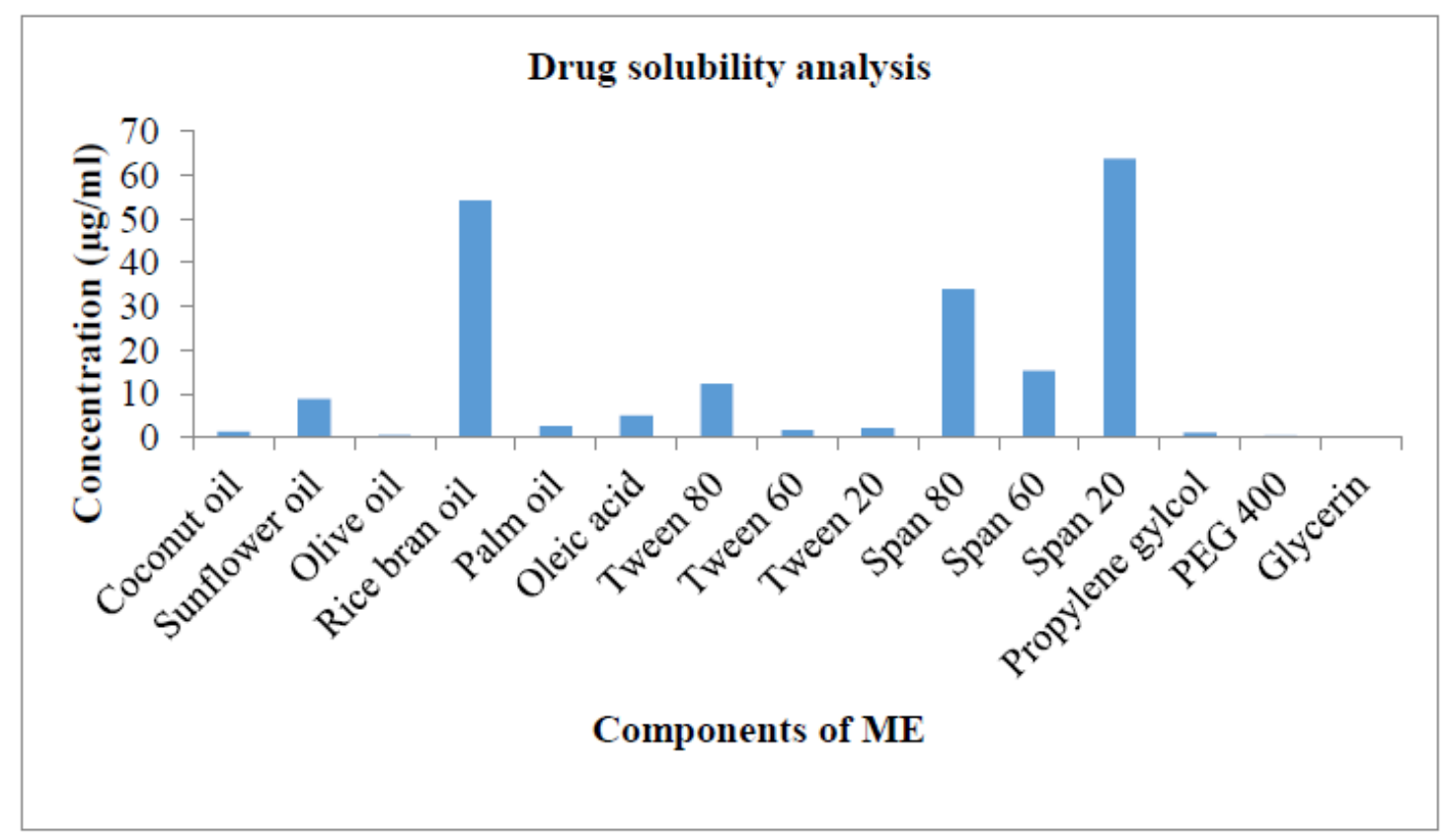

FIG 1: Solubility of MN in various solvents saturated for $24 \mathrm{~h}$ at room temp

Therefore, we formulated ME using Rice bran oil and Sunflower oil as an oil, propylene glycol as a cosurfactant and used tween 80 and span 20 for use as surfactants. 


\subsection{Pseudoternary phase diagram}

The determination of the ratio of various surfactants and co surfactants is a critical aspect of microemulsion formulation design. The most common and effective method for screening of surfactant and cosurfactant proportions is the construction of pseudoternary phase diagrams. Water titration method was employed in the study because this method is economical and widely used. In a pseudoternary phase diagram, each corner of the diagram represents $100 \%$ of any particular component. According to the pseudoternary phase diagrams, a larger region of microemulsion formation was observed at a Smix of 1:1 Smix of $1: 2$ than at a 2:1. Therefore, a Smix of $1: 1$ and $1: 2$ was selected for further formulation of MEs.

\subsection{Preliminary trial batches for optimization of oil and Smix concentration range}

By trial and error method, the concentration range of oil and Smix was determined based on water uptake in the formulation and the \% transparency. The \% w/w of oil and Smix was optimized based on the considerations that high concentration of surfactant could cause toxicity and skin irritation.

\subsection{Results for ME evaluation}

The results of characterization for different parameters are given in Table 2 respectively. It was observed that the disperse system of eight formulations of ME were macroscopically identical i.e., homogenous.
The $\mathrm{pH}$ of all $\mathrm{MN}$ ME was ranged between $3.51 \pm 0.20$ and $3.78 \pm 0.03$. However, MEs had very low viscosity to retain at the affected position sustainably; the viscosity played an important role in promoting the drug retention in the stratum corneum and viable epidermis. Transmittance of all ME were determined to know the effectiveness of transmitting the radiant energy.

The globule size of different MN ME was found to be in the range $290.90 \pm 4.42 \mathrm{~nm}$ to $1177.00 \pm 32$. Polydispersity index is a measure of particle homogeneity and it varied from $0.17 \pm 0.16$ to $0.67 \pm 0.02$. It signifies particle in ME had narrow size of distribution. The zeta potential is a key indicator of the state of the particle surface, stability and predicting the long term stability of colloidal dispersions. The zeta potential of all MN ME ranged between $-50.03 \pm 0.49$ and $-13.73 \pm 0.49$. The results indicated that the prepared MEs could meet all the specifications which are suitable for topical application.

The drug content was found to be in a range $72.00 \pm 0.523 \%$ and $84.21 \pm 0.445 \%$ respectively among all the MEs formulations. The incorporation of miconazole in microemulsion formulations led to enhancement of the drug release profile depicted in Figure 2 by allowing constant and regular in vitro release as well as reducing particle size to that suited to a microemulsion. It was observed that maximum drug release from microemulsion was achieved within $8 \mathrm{~h}$.

\begin{tabular}{|c|c|c|c|c|c|c|c|}
\hline Code & $\begin{array}{l}\text { pH } \quad \pm \\
\text { SD* }\end{array}$ & $\begin{array}{l}\text { Viscosity } \pm \text { SD }^{*} \\
(\mathrm{cps})\end{array}$ & $\begin{array}{l}\% \\
\text { transmittance } \pm \\
\text { SD }\end{array}$ & $\begin{array}{lll}\text { Globule } & \text { size } \quad \pm \\
\text { SD* }(\mathrm{nm}) & & \end{array}$ & $\begin{array}{l}\text { PDI } \pm \\
\text { SD* }\end{array}$ & $\begin{array}{l}\text { Zeta potential } \pm \\
\text { SD* }(\mathrm{mV})\end{array}$ & $\begin{array}{l}\text { Drug content } \pm \\
\text { SD* }\end{array}$ \\
\hline $\mathrm{F} 1$ & $4.10 \pm 0.73$ & $589.13 \pm 0.46$ & $0.060 \pm 0.05$ & $483.87 \pm 30.25$ & $0.54 \pm 0.13$ & $-43.50 \pm 0.90$ & $73.94 \pm 0.254$ \\
\hline $\mathrm{F} 2$ & $3.51 \pm 0.20$ & $466.93 \pm 4.10$ & $0.104 \pm 0.03$ & $290.90 \pm 4.42$ & $0.20 \pm 0.04$ & $-15.33 \pm 0.55$ & $84.21 \pm 0.445$ \\
\hline F3 & $5.21 \pm 0.09$ & $494.37 \pm 10.0$ & $0.166 \pm 0.15$ & $631.50 \pm 123.31$ & $0.38 \pm 0.23$ & $-43.77 \pm 0.83$ & $72.82 \pm 0.789$ \\
\hline $\mathrm{F} 4$ & $3.78 \pm 0.03$ & $143.23 \pm 1.27$ & $0.254 \pm 0.07$ & $873.00 \pm 170.78$ & $0.17 \pm 0.16$ & $-13.73 \pm 0.49$ & $83.14 \pm 0.365$ \\
\hline F5 & $4.61 \pm 0.13$ & $665.63 \pm 1.12$ & $0.385 \pm 0.15$ & $1177.00 \pm 32.97$ & $0.46 \pm 0.03$ & $-50.03 \pm 0.49$ & $72.00 \pm 0.523$ \\
\hline F6 & $5.28 \pm 0.05$ & $173.73 \pm 2.40$ & $0.248 \pm 0.03$ & $751.63 \pm 27.11$ & $0.42 \pm 0.18$ & $-22.23 \pm 0.37$ & $73.18 \pm 0.623$ \\
\hline F7 & $4.56 \pm 0.22$ & $282.70 \pm 0.70$ & $0.362 \pm 0.21$ & 443.93 .98 & $0.34 \pm 0.28$ & $-46.43 \pm 0.80$ & $78.21 \pm 0.854$ \\
\hline $\mathrm{F} 8$ & $4.89 \pm 0.22$ & $62.23 \pm 1.27$ & $0.166 \pm 0.08$ & $424.53 \pm 45.13$ & $0.67 \pm 0.02$ & $-19.93 \pm 0.40$ & $74.80 \pm 0.783$ \\
\hline
\end{tabular}




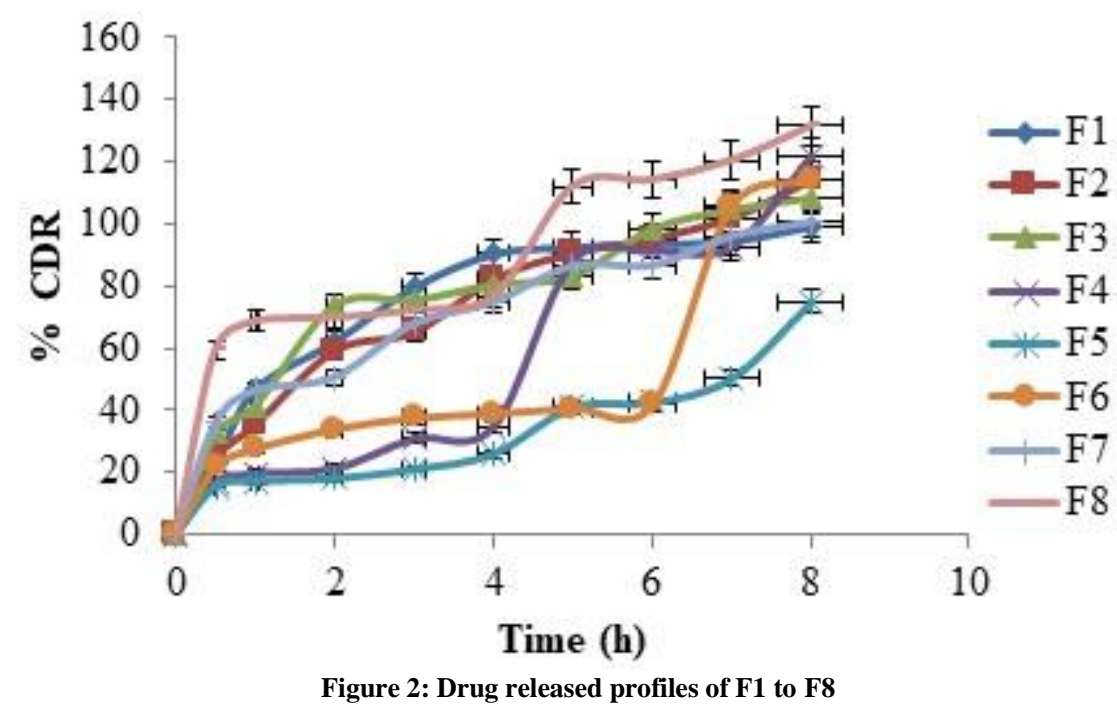

\subsection{Formulation optimization}

The ratio of surfactant and cosurfactant was determined using pseudoternary phase diagrams. However, the ratio of oil, water, and surfactant that enables microemulsion formation must also be determined. $2^{3}$ factorial designs is the most common method for optimization. A $2^{3}$ factorial designs consists of 3 factors and 2 levels. 8-run experiments were designed to study the influence of twofactor, two-level on the response variables and the response data were recorded. The In this research, the contents of oil (X1), surfactant (X2) and $\mathrm{S}_{\operatorname{mix}}(\mathrm{X} 3)$ were chosen as the three independent variables. Application of Design of Experiment (DOE), the relationship between the three independent variables and each response was observed. The main effect as well as the interaction effect was studied for all the three variables. As seen from the 3D surface graph obtained through design expert software, $X$ axis represents the responses.

The coded equation for the particle size as per DOE is represented as follows:

Particle size $=634.54+64.72(\mathrm{~A})-49.53(\mathrm{~B})$ 41.31(C)-61.67(AB)-

223.74(AC)+105.05(BC)

The p-value obtained from DOE was found to be significant i.e. 0.0247 and the $\mathrm{r}^{2}$ value was found to be 0.9998 .
The coded equation for the Zeta potential as per DOE is represented as follows: Zeta potential $=-31.87$ 2.79(A)+14.06(B)+0.9050(C)

The $\mathrm{p}$-value obtained from DOE was found to be significant i.e. $<0.0001$ and the $\mathrm{r}^{2}$ value was found to be 0.9965 .

The coded equation for the drug release as per DOE is represented as follows:

Drug release $=87.80+3.41(\mathrm{~A})+1.95(\mathrm{~B})-$ $0.9875(\mathrm{C})-0.1750(\mathrm{AB})-2.19(\mathrm{AC})-$ $0.1375(\mathrm{BC})$

The p-value obtained from DOE was found to be significant i.e.0.0152 and the $\mathrm{r}^{2}$ value was found to be 0.9999 .

Where A is oil, B is the surfactant, $\mathrm{C}$ is the $\mathrm{S}_{\operatorname{mix}}$ ratio, $\mathrm{AB}$ is the interaction between oil and surfactant, $\mathrm{AC}$ is the interaction between oil and $\mathrm{Smix}$ ratio, $\mathrm{BC}$ is the interaction between surfactant and Smix.

The above model equation carries factors along with coefficients both positive and negative, which quantify response values. Synergistic effects are indicated by positive sign while antagonistic effects are indicated by negative sign. The $\mathrm{p}$ value is $<0.05$ respectively for all the three responses, indicated that the polynomial regression equations were statistically significant. The $3 \mathrm{D}$ response surface plots 
of independent variables and response variables were shown in Fig. 3.
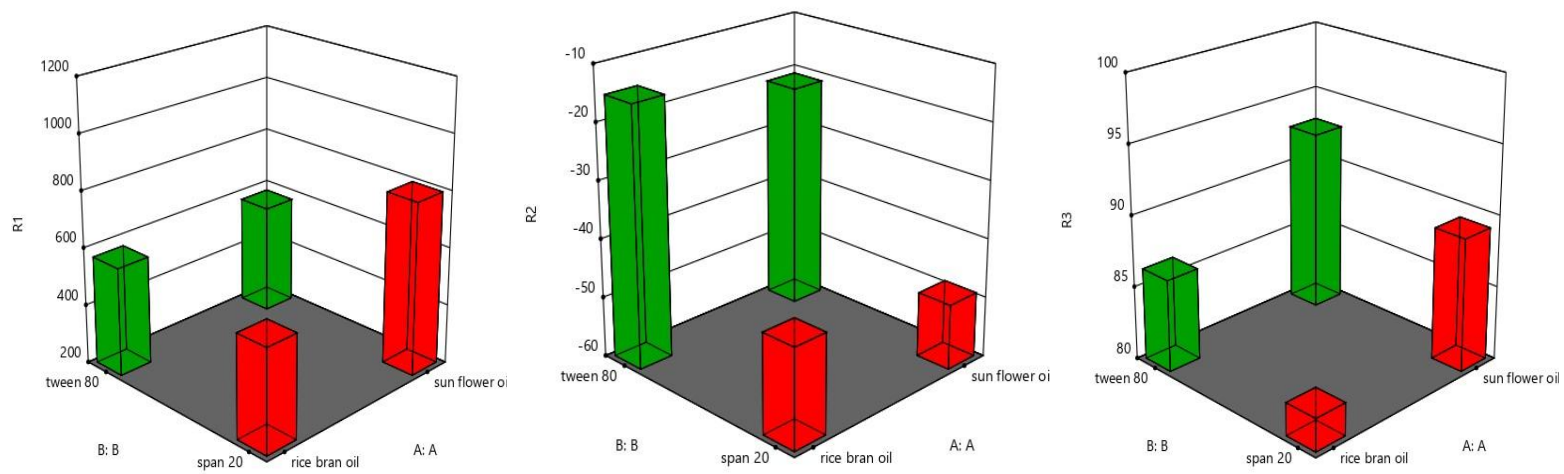

Fig No. 3: 3D surface plot of a) Globule size; b) Zeta potential; c) Drug release

After applying the Design-Expert ${ }^{\circledR}$ Software 12.0 for the optimization of final batch ME, the formulation containing rice bran oil, tween 80 at $1: 2$ Smix ratio was selected as optimized ME due to high desirability. The design space which was used for optimization includes 450nm particle size, $-35 \mathrm{mV}$ zeta potential, $90 \%$ drug release. Among the six different solutions given by the software during optimization, the working space composition for optimized formulation was selected i.e., $\mathrm{X} 1$ as Rice bran oil, X2 as Tween $80, \mathrm{X} 3$ being 1:2 Smix ratio owing to the high desirability of 0.837 .

\subsection{Fitting of data to the model}

The optimized formulation was prepared and evaluated which showed the results within the desirability range as average globule size of $460 \mathrm{~nm}$ with the PDI of $0.280,-44.4 \mathrm{mV}$ of zeta potential and $96.33 \%$ of drug release at the end of $8^{\text {th }}$ h.

\subsection{Preparation of microemulsion-based gel}

The polymers TSP and carbopol 934 was selected as the suitable polymer for the formulation of microemulsion-based gel. As the viscosity of TSP and CP 934 was highest among other polymer concentrations, $1 \%$ concentration was selected as the suitable one for topical application.

\subsection{Evaluation of microemulsion-based hydrogel}

MBG was a semi-solid gel with a translucent appearance, and no phase separation occurred under centrifugation at $3000 \mathrm{rpm}$ for $15 \mathrm{~min}$ at $4{ }^{\circ} \mathrm{C}$. Various evaluation parameters of optimized $\mathrm{MBG}$ of TSP and CP are shown in Table 3. The $\mathrm{pH}$ value of MBG of TSP was $6.51 \pm 0.20$ and of $\mathrm{MBG}$ of $\mathrm{CP}$ was $6.10 \pm 0.73$ approximating the $\mathrm{pH}$ value of normal skin, which implied its compatibility with skin. Viscosity was determined by using T-bar spindle $(96 \mathrm{~F})$ at $50 \mathrm{rpm}$. The viscosity of MBG was significantly higher than that of PAB ME.

The average droplet size of MBG of TSP was $290.90 \pm 4.42 \mathrm{~nm}$ with PDI of $0.20 \pm 0.04$ and $\mathrm{MBG}$ of $\mathrm{CP}$ was $483.87 \pm 30.25 \mathrm{~nm}$ with PDI of $0.54 \pm 0.13$. The addition of gelling agent caused a slight increase in globule size. The results indicated that ME droplets aggregated partly during gelation process. It can be deduced that the interfacial tension between oil and water of ME increases slightly owing to the addition of gelling agent, then some ME globules aggregate when they are entrapped within the gel network. MBG of TSP shows lesser particle size, higher zeta potential and more homogeneous formulation than compared to that of $\mathrm{MBG}$ of $\mathrm{CP}$. The negative zeta potential indicates that globules of microemulsion had no charge, that is, the system was stable. As there 
was no charge on globules, no flocculation of globules occurred and hence, microemulsion was found to be stable.

Spreadability coefficient of MBG of TSP and MBG of CP was found to be 8.36 $\mathrm{gcm} / \mathrm{sec}$ and $9.3 \mathrm{gcm} / \mathrm{sec}$. The ex vivo bioadhesive strength was found to be 5 $\mathrm{kg} / \mathrm{cm}^{2}$ and $3 \mathrm{~kg} / \mathrm{cm}^{2}$ for MBG of TSP and $\mathrm{CP}$ respectively. The $\mathrm{MN}$ content in the optimized MBG formulation of TSP and CP was found to be $84.21 \%$ and $73.94 \%$. \% CDR at the end of $8 \mathrm{~h}$ was $87.89 \%$ for TSP and $82.13 \%$ for CP. The other parameters of MBGs and marketed brand were reported in Table 4.

Kinetic modeling and mechanism of drug release

The correlation coefficient $\left(\mathrm{R}^{2}\right)$ of the zero order model was found to be 0.923 , slightly higher when compared with the other model for final selected optimized batch of MBG. Hence the release of drug from the preparation followed zero order kinetics.

In vitro drug release studies were carried out for $8 \mathrm{~h}$ for both MBGs and commercial gel. The permeation ability of the both the MBG was evaluated using the ex vivo permeation studies. The permeation profiles of the tested MBGs and standard formulation through excised abdominal skins of rat are shown in Table 4. A steady increase of $\mathrm{MN}$ in the receptor chambers with time was observed. The permeation profiles of MBG followed zero order release kinetics. All the MBG showed that percent of drug permeated throughout $8 \mathrm{~h}$ higher than the saturated solution of $\mathrm{MN}$. MN had only a low accumulative amount of $\mathrm{MN}$ permeated about $35.7 \%$ at 8 h.MBG of TSP and CP enhanced the ex vivo drug diffusion penetration of the MN. MBG was not effective compared to $\mathrm{MN} \mathrm{ME}$, the release of the drug did not show significant increase on the application of MBG

Table 3: Evaluation parameters of optimized microemulsion based gel

\begin{tabular}{|c|c|c|}
\hline Evaluation parameter & TSP MBG gel & CP MBG gel \\
\hline $\mathrm{pH} \pm \mathrm{SD}^{*}$ & $6.51 \pm 0.28$ & $6.10 \pm 0.13$ \\
\hline Viscosity \pm SD $^{*}$ (cps) & $466.93 \pm 4.10$ & $589.13 \pm 0.46$ \\
\hline Globule size $\pm \mathrm{SD}^{*}(\mathrm{~nm})$ & $290.90 \pm 4.424$ & $483.87 \pm 30.25$ \\
\hline $\mathrm{PDI} \pm \mathrm{SD} *$ & $0.20 \pm 0.047$ & $0.54 \pm 0.13$ \\
\hline Zeta potential $\pm \mathrm{SD}^{*}(\mathrm{mV})$ & $-43.50 \pm 0.90$ & $-15.33 \pm 0.55$ \\
\hline Transmittance $\pm \mathrm{SD}^{*}$ & $0.1043 \pm 0.03$ & $0.0600 \pm 0.05$ \\
\hline Drug content $\pm \mathrm{SD}^{*}(\%)$ & $87.29 \pm 0.34$ & $83.34 \pm 0.69$ \\
\hline Spreadability coefficient \pm SD* $(\mathrm{gcm} / \mathrm{s})$ & $8.36 \pm 1.37$ & $9.3 \pm 2.44$ \\
\hline Ex vivo bioadhesive strength $\left(\mathrm{kg} / \mathrm{cm}^{2}\right)$ & 5 & 3 \\
\hline Ex vivo skin permeation \pm SD* $(\%)$ & $84.21 \pm 3.75$ & $73.94 \pm 4.64$ \\
\hline $\mathrm{CDR} \pm \mathrm{SD}^{*}(\%)$ & $87.89 \pm 1.67$ & $82.13 \pm .83$ \\
\hline
\end{tabular}

Table 4: Evaluation of MBGs and 2\% Miconazole gel

\begin{tabular}{|l|l|l|l|}
\hline Evaluation parameters & MBG TSP & MBG CP & 2\% Miconazole gel \\
\hline $\mathrm{J}_{\mathrm{Ss}} \pm \mathrm{SD}^{*}\left(\mathrm{mg} / \mathrm{cm}^{2} \mathrm{~min}\right)$ & $0.289 \pm 0.73$ & $0.298 \pm 0.83$ & $0.231 \pm 0.46$ \\
\hline $\mathrm{K} \mathrm{p} \pm \mathrm{SD}^{*}(\mathrm{~cm} / \mathrm{min})$ & $0.057 \pm 0.35$ & $0.059 \pm 0.25$ & $0.046 \pm 0.64$ \\
\hline $\mathrm{E}_{\mathrm{r}} *^{*} \pm \mathrm{SD}^{*}$ & $1.25 \pm 0.36$ & $1.29 \pm 0.46$ & 1 \\
\hline \multicolumn{4}{|c}{$*_{n=3} *^{*}$ respect to $\%$ miconazole gel }
\end{tabular}

The total scores for irritation test in control group and MBG of TSP was found to be zero whereas in $\mathrm{MBG}$ of $\mathrm{CP}$ showed a skin irritation score of 0.83 . MBG of TSP favored the formulation administered and spread over the skin, and sustainably retained on the skin, offering a long-last action and a potential target to treat skin diseases. MN MBG of TSP did not have skin irritation whereas MBG of CP showed irritation. It is evaluated that the TSP MBG is not a skin irritant. This result revealed that all excipients used in MBG TSP are safe for topical application. Therefore, it may be considered that formulation has no irritation potential and safe for skin.

The optimized MBG (having 1\% polymer concentration) showed the sustained release of $\mathrm{MN}$ compared with MEs. 
Results of in vitro antifungal activity are shown in Fig 4. MBG of TSP has shown comparable antifungal potency using Cryptococcus neoformans whereas exhibited superior potential in Candida albicans when compared to marketed formulations. MBG of $\mathrm{CP}$ exhibited inferior antifungal activity in comparison to marketed miconazole gel. There was considerable difference between the zones of inhibition of MBG of TSP and MBG of $\mathrm{CP}$. The possible justification for improved activity was the synergistic action of miconazole and TSP in the MBG of TSP.
These results prove that MBG of TSP systems were with maximum fungicidal efficacies due to its bioadhesiveness and higher drug loading capacity which resulted in significant penetration of miconazole into fungal cell membranes. Therefore, MBG of TSP were found to be superior in binding to CYP450 dependent 14- $\alpha$ sterol demethylase and inhibiting ergosterol synthesis in fungal cell wall. Thus, MBG of TSP systems had shown superior antifungal potentials in comparison with standard drug, $\mathrm{ME}$ and MBG of CP.

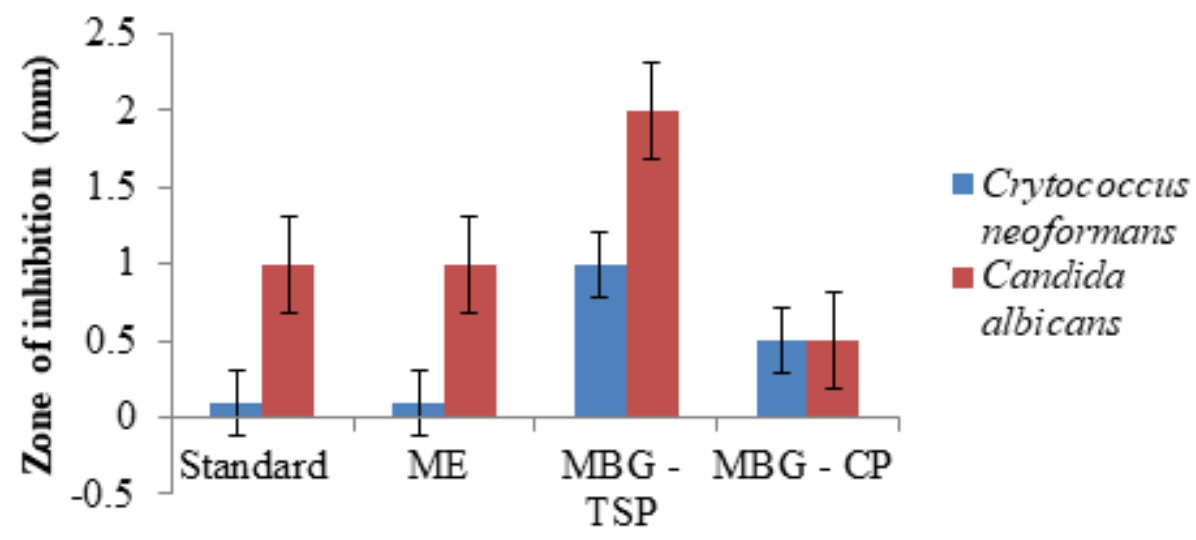

Figure 4: Antifungal study

\section{Statistical analysis}

The data obtained for different formulations was analysed by one way ANOVA. The values were considered to be statistically significant when the $P$ value $\leq 0.05$. It was observed that the $\mathrm{P}$ value of all responses for linear model was found to be $<0.05$. Hence, the results obtained considered as significant.

\section{Stability study}

Stability testing was carried out for MBG of TSP and various evaluation parameters of optimized MBG after 3 months interval at various temperatures showed a slight variation in the values which could not affect the stability of the period during its shelf life as mentioned in Table 5.

Table 5: Stability parameters of optimized microemulsion based gel
\begin{tabular}{|l|l|l|l|l|l|}
\hline Temperature $\left({ }^{\circ} \mathbf{C}\right)$ & $\begin{array}{l}\text { Globule size } \\
\pm \text { SD } *(\mathbf{n m})\end{array}$ & PDI \pm SD $^{*}$ & $\begin{array}{l}\text { Zeta potential } \\
\pm \text { SD }(\mathbf{m V})\end{array}$ & $\%$ Drug content \pm SD $*$ & $\begin{array}{l}\% \text { CDR } \pm \\
\text { SD }^{*}\end{array}$ \\
\hline $\begin{array}{l}4^{\circ} \mathrm{C} \\
(\text { Refrigerator })\end{array}$ & $463.56 \pm 4.6$ & $0.48 \pm 0.24$ & $-47.64 \pm 0.38$ & $67.46 \pm 3.76$ & $73.75 \pm 2.94$ \\
\hline $\begin{array}{l}\left(30^{\circ} \mathrm{C}\right) \\
\text { Room temperature }\end{array}$ & $412.84 \pm 3.5$ & $0.56 \pm 0.65$ & $-41.89 \pm 0.56$ & $83.59 \pm 3.96$ & $82.68 \pm 1.84$ \\
\hline $\begin{array}{l}45^{\circ} \mathrm{C} \\
(\text { Hot air oven })\end{array}$ & $397.63 \pm 4.5$ & $0.27 \pm 0.83$ & $-36.94 \pm 0.64$ & $86.80 \pm 4.82$ & $83.23 \pm 2.46$ \\
\hline
\end{tabular}

\section{CONCLUSION}

The formulation of miconazole nitrate microemulsion based bioadhesive gel was developed by employing factorial design. Microemulsion was prepared with Rice bran oil, Tween 80 and PG to achieve 
the optimum response of globule size, zeta potential and drug release. Viscosity and retention on the skin was achieved by employing the bioadhesive gels like $1 \%$ of TSP and carbopol 934. Following ex vivo permeation study on rat skin, the final formulation of MBG of TSP showed higher permeation as compared to conventional gel preparation which could contributes to the enhanced transdermal ability. Anti fungal activity study supports the better performance of MBG of TSP compared to commercial formulation. Therefore, the results indicated that $\mathrm{MN}$ MBG of TSP could be a promising formulation in drug delivery than the conventional topical dosage forms.

Acknowledgement: The authors are thankful to Acharya \& BM Reddy college of Pharmacy, Karnataka, Bengaluru for providing the necessary facilities.

Conflict of Interest: The authors report no conflicts of interest in this work.

\section{Source of Funding: None}

\section{Ethical Approval: Approved}

\section{REFERENCES}

1. Kaur, I.P.; and Kakkar, S. Topical delivery of antifungal agents. Exp. Opin. Drug Del. 2010, 7(11), 1303-1327. doi:10.1517/17425247.2010.525230

2. Ameen M. Epidemiology of superficial fungal infections. Clin Dermatol. 2010, 28(2), 197- 201.

3. Vermand, P.; Pathak, K. Nanosized ethanolic vesicles loaded with econazole nitrate for the treatment of deep fungal infections through topical gel formulation. Nanomed. 2012, 8(4), 489-496.

4. Seh, H.S.; Kyung, M.L.; Jong, B.K.; Sang, G.L.; Myung, J.K.; and Young, W.C. Improved skin delivery of voriconazole with a nanostructured lipid carrier-based hydrogel formulation. Chem. Pharm. Bull. 2014, 62(8), 793-798.

5. Nastiti, C.; Ponto, T.; Abd, E.; Grice, J.; Benson, H.; Roberts, M. Topical nano and microemulsions for skin delivery. Pharmaceutics. 2017, 9(4), 37.

6. Tejada, G.; Lamas, M.C.; Sortino, M.; Alvarez, V.A.; and Leonardi, D. Composite Microparticles Based on Natural Mucoadhesive Polymers with Promising Structural Properties to Protect and Improve the Antifungal Activity of Miconazole Nitrate. AAPS Pharm Sci Tech. 2018, 19, 3712-3722.

7. Santos, P.; Watkinson, A.C.; Hadgraft, J.; Lane, M.E. Application of microemulsions in dermal and transdermal drug delivery. Skin Pharmacol. Physiol. 2008, 21, 246259.

8. Anton, N.; Vandamme, T.F. Nanoemulsions and micro-emulsions: Clarifications of the critical differences. Pharm. Res. 2011, 28, 978-985.

9. Zheng, W.; Hong, J.M.; Xue, M.Z.; Peng, K.M.; Sheng, N.L.; Feng, P.Z. Lower irritation microemulsion-based rotigotine gel: formulation optimization and in vitro and in vivo studies. Int. J. Nanomed. 2015, 10,633-644. doi:10.2147/ijn.s74079.

10. Kailas, K.M.; Shashikant, C.D.; Remeth, J.D. Microemulsion based bioadhesive gel of itraconazole using tamarind gum: in vitro and ex vivo evaluation. Marmara Pharm. J. 2017, 21(3), 688-700. DOI: 10.12991/marupj.323593.

11. Mrunali, R.P.; Rashmin, B.P.; Jolly, R.P.; and Bharat, G.P. Novel microemulsionbased gel formulation of tazarotene for therapy of acne. Pharm. Dev. Tech. 2015, 21, 921-932.

12. Loftsson, T. Drug Degradation in Semisolid Dosage Forms in Drug Stability for Pharmaceutical Scientists. 2014, pp. 105$107 . \quad$ doi:10.1016/b978-0-12-4115484.00004-0

13. Vazquez, J.A.; Sobel, J.D. Miconazole mucoadhesive tablets: a novel delivery system. Clin. Infect. Dis. 2012, 54, 14801484.

14. Zhang, L.W.; Fu, J.Y.; Hua, H.; Yan, Z.M. Efficacy and safety of miconazole for oral candidiasis: a systematic review and metaanalysis. Oral Diseases. 2016, 22, 185-195. doi:10.1111/odi.12380.

15. Amaral, A.C.; Saavedra, P.H.V.; Oliveira S.; Melo, M.T.; Tedesco, A.C.; Morais, P.C.; Bocca, A.L. Miconazole loaded chitosan-based nanoparticles for local treatment of vulvovaginal candidiasis fungal 
infections. Colloids and Surfaces B: Biointerfaces. 2019, 174, 409-415. doi:10.1016/j.colsurfb.2018.11.048

16. Moody, M.R.; Young, V.M.; Morris, M.J.; \& Schimpff, S.C. In vitro activities of miconazole, miconazole nitrate, and ketoconazole alone and combined with rifampin against Candida spp. and Torulopsis glabrata recovered from cancer patients. Antimicrob. Agents Chemother. 1980, 17(5), 871-875. doi:10.1128/aac.17.5.871.

17. Isham, N.; \& Ghannoum, M.A. Antifungal activity of miconazole against recent Candida strains. Mycoses. 2010, 53(5), 434-437. doi:10.1111/j.14390507.2009.01728.x

18. Cao, M.; Ren, L.; Chen, G. Formulation Optimization and Ex Vivo and In Vivo Evaluation of Celecoxib MicroemulsionBased Gel for Transdermal Delivery. AAPS PharmSciTech. 2017, 18(6), 1960-1971.

19. Vadlamudi, H. C.; Yalavarthi, P. R.; M V, B. R.; Rasheed, A.; and N, Tejeswari. In vitro characterization studies of selfmicroemulsified bosentan systems. Drug Dev. Ind. Pharm. 2017, 43(6), 989-995. https://doi.org/10.1080/03639045.2017.128 7720

20. Thakur, N.K.; Bharti, P.; Mahant, S.; Rao, R. Formulation and characterization of benzoyl peroxide gellified emulsions. Sci. Pharm. 2012, 80, 1045-1060.

21. Chudasama, A.; Patel, V.; Nivsarkar, M.; Vasu, K.; Shishoo, C. Investigation of microemulsion system for transdermal delivery of itraconazole. J. Adv. Pharm. Technol. Res. 2011, 2, 30-38.

22. Bachhav, Y.G.; Patravale, V.B. Microemulsion based vaginal gel of fluconazole: formulation, in vitro and in vivo evaluation. Int. J. Pharm. 2009, 365(12), 175-179.

23. Shinde, U.A.; Modani, S.H.; Singh, K.H. Design and development of repaglinide microemulsion gel for transdermal delivery. AAPS PharmSciTech. 2018, 19(1), 315325.

24. Chauhan, T.; Parashar, B.; Arora, S. Design and evaluation of diclofenac sodium gel. Int. J. Pharm. Chem. Sci. 2013, 2(1), 72-81.
25. Gungor, S.; Bergisadi, N. In vitro release studies on topical gel formulation of nimesulide. Pharmazie. 2003, 58(2),155156.

26. Prasanna, R.Y.; Hyndavi, N.; Harini, C.V.; Rajesh, S.N.; Jamal, B.D.; \& Tejeswari, N. In vitro assessment of non-irritant microemulsified voriconazole hydrogel system. Artif. Cells Nanomed. Biotechnol. 2016, 45, 1539-1547.

27. Sujith, S.N.; Molly, M.; and Sreena, K. Formulation and evaluation of herbal cream containing curcuma longa. Int. J. Pharm. Chem. Sci. 2012, 1(4), 1362 -1368.

28. Lalit, K.; And Ruchi, V. Chemical stability studies of bioadhesive topical gel. Int. J. Pharm. Pharm. Sci. 2011, 1(3), 101-104.

29. Sabale, V.; Vora, S. Formulation and evaluation of microemulsion-based hydrogel for topical delivery. Int. J. Pharm. Investig. 2012, 2(3), 140-149.

30. Ashara, K.C.; Paun, J.S.; Soniwala, M.M.; Chavda, J.R. Microemulgel of voriconazole: an unfathomable protection to counter fungal contagiousness. Folia Med. 2017, 59(4), 461- 471.

31. Yogeshwar, G.B.; Vandana, B.P. Formulation of meloxicam gel for topical application: In vitro and in vivo evaluation. Acta Pharm. 2010, 60, 153-16.

32. Kaewbanjong, J.; Amnuaikit, T.; Souto, E.B.; Boonme, P. Antidermatophytic Activity and Skin Retention of Clotrimazole Microemulsion and Microemulsion-Based Gel in Comparison to Conventional Cream. Skin Pharmacol. Physiol. 2018, 31(6), 292297.

33. Ashara, K.C.; Paun, J.S.; Soniwala, M.M.; Chavda, J.R.; Mendapara, V.P.; Mori, N.M. Microemulgel: an overwhelming approach to improve therapeutic action of drug moiety. Saudi Pharm. J. 2016, 24(4), 452457.

How to cite this article: Abhirami Venkatachalam, Harini Chowdary Vadlamudi, Sneha Bharti. Factorial design executed development of miconazole nitrate microemulsion based bioadhesive gel and its evaluation. International Journal of Research and Review. 2022; 9(1): 574-587. DOI: https:// doi.org/10.52403/ijrr.20220167 